Relación del proveedor de servicios farmacéuticos con sus clientes: Una mirada desde el Marketing Relacional en el departamento de Sucre

\title{
RELACIÓN DEL PROVEEDOR DE SERVICIOS FARMACÉUTICOS CON SUS CLIENTES: UNA MIRADA DESDE EL MARKETING RELACIONAL EN EL DEPARTAMENTO DE SUCRE
}

\section{RELATIONSHIP BETWEEN PHARMACEUTICAL SERVICES PROVIDER AND CUSTOMERS: A VIEW FROM THE RELATIONSHIP MARKETING IN THE DEPARTMENT OF SUCRE.}

RECEPCIÓN: Febrero 3 de 2014

John Pablo Martínez Benítez*

Universidad de Sucre, Sincelejo-Colombia

ACEPTADO: Abril 3 de 2014

\section{RESUMEN}

El presente artículo de investigación se realizó con el objetivo de analizar la relación del proveedor de servicios farmacéuticos con sus clientes, desde el marketing relacional en el departamento de Sucre (Colombia). El estudio se fundamentó en la perspectiva del paradigma interpretativo, y se utilizó el método de investigación descriptivo, basado en la estructura de investigación de Quivy y Campenhoudt, que se articuló con las etapas del procedimiento científico. Entre los resultados obtenidos, se destaca la identificación de tres tipos de clientes en el sector farmacéutico. Además, se encontró que los proveedores de este sector en Sucre han generado fuertes lazos con sus clientes, a pesar de que los canales de comunicación con estos son insuficientes. Los proveedores farmacéuticos también han generado alianzas con los distribuidores mayoristas para satisfacer las necesidades de los clientes. Se concluyó que para qué la relación del sector farmacéutico con sus clientes perdure, se requiere brindar un servicio que inspire confianza y le proporcione al cliente la seguridad de que el producto que se le ofrece llena sus expectativas.

Palabras clave: Marketing de relaciones, calidad, satisfacción, Sector Farmacéutico

Este artículo se puede referenciar:

Martínez, J.P. (2014). Relación del proveedor de servicios farmacéuticos con sus clientes: una mirada desde el marketing relacional en el departamento de Sucre. Desarrollo Gerencial Revista de la Facultad de Ciencias Económicas, Administrativas y Contables. Universidad Simón Bolívar, Vol. 6 (1) P.196-231.

\footnotetext{
*Administrador de Empresas, vinculado a la universidad de Sucre-Colombia. Correo electrónico: jhon.martinez@unisucre.edu.co
} 
Relación del proveedor de servicios farmacéuticos con sus clientes: Una mirada desde el Marketing Relacional en el departamento de Sucre

\section{ABSTRACT}

This research paper, whose aim was to analyze the relationship between pharmaceutical services provider and customers from the relationship marketing in Sucre, Colombia. The study was based on the perspective of the interpretive paradigm and the research method used was descriptive, based on the research structure of Quivy and Campenhouldt, articulated with the stages in the scientific procedure. Among the results obtained, three types of clients within the pharmaceutical sector were identified; besides, providers of the Pharmaceutical sector in Sucre have created strong bonds with their customers, despite the fact that channels of communication with them are

insufficient. Pharmaceutical providers have also created partnerships with wholesale distributors of the Pharmaceutical sector to meet the customers' needs. It was concluded that for the relationship between the Pharmaceutical sector and clients' lasts, it requires to provide a service that inspires confidence and assure them that the product offered will meet their expectations.

Key words: Marketing, Relationships, Quality, satisfaction, Pharmaceutical sector 
Relación del proveedor de servicios farmacéuticos con sus clientes: Una mirada desde el Marketing Relacional en el departamento de Sucre

INTRODUCCIÓN

En cada sector de la economía, resulta obvio el establecimiento de vínculos que permiten satisfacer las necesidades del cliente (Walter et al, 2001; Morgan y Hunt, 1994; Concha y Solikova, 2000). Y es que siempre está en juego la posibilidad de mantener los beneficios sociales, entre los que no solo se cuenta el apoyo de quienes establecen la relación, sino a todos aquellos que se benefician directamente con los productos resultantes de tal relación. En este sentido, Concha y Solikova (2000) afirman que la confianza en la empresa proveedora, o en el vendedor que la representa, influye en la interacción futura del comprador y en la cooperación con el proveedor.
Es claro que el modelo de marketing tradicional ha cambiado, y se ha transformado en una nueva filosofía que postula el establecimiento de relaciones cercanas y a largo plazo, basadas en altos niveles de cooperación y acción conjunta, como un riesgo que puede asegurar ventajas mutuas y satisfacción (Llorens et al, 2006). Así, las nuevas prácticas en la disciplina de dirección de empresas se centran en el sostenimiento de relaciones estratégicas con un número limitado de clientes, y en la planificación de objetivos conjuntos como un camino para alcanzar una ventaja competitiva.

Sin embargo, en el departamento de Sucre, la aplicación de estrategias como elementos integradores de marketing de relaciones en las empresas 
Relación del proveedor de servicios farmacéuticos con sus clientes: Una mirada desde el Marketing Relacional en el departamento de Sucre

del sector farmacéutico es cada vez más crítica, aun cuando estas estrategias influyen en la relación proveedor-cliente, toda vez que los clientes desean ir más allá de la simple transacción. Dichos clientes busca una calidad en el servicio que se le ofrece, así como en la relación con su proveedor inmediato hasta el punto de el cliente se sienta satisfecho y así se genere valor a la empresa.

En la actualidad, la calidad del servicio y de la relación son dos de los factores competitivos más importantes para las empresas. Sandoval (2002) los considera incluso los pilares que permiten el avance y el fortalecimiento de las relaciones con el cliente. De esta manera, se trasciende la idea que ve el servicio como un simple elemento de la logística de la empresa. Ciertamente, es imprescindible percibir el servicio a la clientela como el resultado de las actividades de logística, pero su importancia se explica en función de la forma en que los productos o servicios se entregan y en la constatación de que los clientes se sientan satisfechos, en especial en términos de fiabilidad y rapidez de la entrega.

Las expectativas del usuario se relacionan con lo que esperan recibir como servicio. Y el conocimiento de estas expectativas propiciará, de parte del proveedor, la satisfacción en la entrega de su producto o en la prestación del servicio solicitado. En este aspecto de la relación proveedor-cliente, la calidad involucra tanto aspectos sobre la funcionalidad y adecuación como aspectos del diseño de los productos y servicios, así como al 
Relación del proveedor de servicios farmacéuticos con sus clientes: Una mirada desde el Marketing Relacional en el departamento de Sucre

proceso de elaboración y/o entrega de estos. Tales condiciones influirán en la satisfacción final del cliente.

La descripción y el análisis de las distintas conceptualizaciones a partir del enfoque de las relaciones, permitirá mostrar, entonces, cómo el proceso de la relación cliente- proveedor influye en la calidad del servicio prestado.

\section{MÉTODO}

\section{Diseño}

Este trabajo se realizó a partir de un diseño cualitativo, dado que en él se describió y analizó la relación proveedorcliente, y el rol que juega la calidad en esta relación fundamentándose en el marketing relacional. Se trabajó según la perspectiva del paradigma interpretativo
(Koetting, 1984), debido a que busca describir el acontecimiento estudiado, y permite hacer una rigurosa descripción contextual de las situaciones que posibilitan la intersubjetividad en la captación de la realidad, a través de una recogida sistemática de los datos sobre los cuales se obtendrán resultados.

La presente investigación utilizó el método descriptivo (Ramírez et al, 2004), puesto que busca describir y analizar los aspectos que influyen en la relación del proveedor de servicios farmacéuticos con sus clientes, a partir del marketing relacional, en el departamento de Sucre.

\section{Participantes}

Los participantes del estudio fueron: las droguerías, los laboratorios farmacéuticos, los distribuidores 
Relación del proveedor de servicios farmacéuticos con sus clientes: Una mirada desde el Marketing Relacional en el departamento de

mayoristas, las cajas de compensación, las cadenas, las entidades prestadoras de salud, los profesionales en la salud, el gobierno (Ministerio de Protección Social, Ministerio de Comercio, Industria y Turismo y sus entidades adscritas) y los consumidores finales del departamento de Sucre, específicamente de su capital, Sincelejo, tomando como muestra aleatoria: 164 droguerías, de las 285 inscritas oficialmente en el departamento de Sucre.

\section{Instrumentos}

Se aplicó la técnica de la entrevista exploratoria expuesta por Quivy y Campenhoudt (2006), la cual permite analizar la relación clienteproveedor- Los cuestionarios fueron diseñados con preguntas abiertas y flexibles de fácil comprensión para los encuestados.

\section{Procedimientos}

Se siguió la estructura de investigación de Quivy y Campenhoudt (2006), articulada con las etapas en los actos del procedimiento científico de la siguiente manera:

La ruptura: En esta etapa de la investigación se trata de articular el caudal "teórico" en lo que respecta al marketing de relaciones. Luego se realiza la exploración de la problemática a través de lecturas que aseguren la calidad del cuestionamiento.

La estructuración del modelo de análisis: Define la estructura teórica que sirve de soporte a la hipótesis de investigación, lo cual posibilita la creación de un 
Relación del proveedor de servicios farmacéuticos con sus clientes: Una mirada desde el Marketing Relacional en el departamento de

constructo conceptual suficiente para los análisis empíricos subsiguientes, y permite analizar las consecuencias obtenidas al final de la comprobación.

La comprobación: A través de la observación, se pone a prueba el análisis empleado, haciendo un análisis cualitativo de las variables que lo integran. Luego se analiza toda la información y se compara lo obtenido en el sector farmacéutico. Finalmente, como resultado de la investigación, se elaboran las conclusiones de la misma, en las cuales se describen los resultados alcanzados.

\section{RESULTADOS}

A partir del estudio de la información proporcionada por el proceso de entrevistas exploratorias, se ha realizó un breve análisis de los resultados obtenidos, a fin de analizar la relación proveedor-cliente en el sector farmacéutico en el departamento de Sucre.

En el mercado de medicamentos, los administradores de las empresas que comercializan fármacos identificaron tres tipos de clientes en el departamento de Sucre, a saber: los clientes directos, aquellos que de acuerdo con sus intereses acuden a las droguerías para obtener un producto de su preferencia que contribuya al mejoramiento de su estado de salud; los clientes que consumen medicamentos con prescripción médica, los cuales provienen del enlace de la cadena de actores del sector farmacéutico: la EPS, ARS o entidad de régimen especial, la droguería 
Relación del proveedor de servicios farmacéuticos con sus clientes: Una mirada desde el Marketing Relacional en el departamento de Sucre

y el consumidor final, en calidad de pacientes pero el pago es cubierto por un tercero; y finalmente, se encuentran los clientes que prefieren acudir con su prescripción a la droguería, dada por médicos particulares y realizar su propio gasto para evitar trámites administrativos ante el tercero.

En este orden de ideas, en el proceso de análisis de la información se observó que muchas droguerías del departamento de Sucre han generado fuertes lazos con sus clientes, entre estas se cuentan: Drogas Económicas, Súper droguerías Olímpicas, Drogas La Rebaja, Droguería La Cascada, Droguerías La Botica, Droguería Súper, Droguería New York, Droguería Inglesa, Droguería El Punto del Diabético, Droguería Ford, Drogas Bucaramanga, Global
Pharmaceutica S.A., Maxifarmas, Distribuciones Hernández \& Cía. Ltda., Drogas La Economía, Droguería La Gran Droguería, Droguería Maxi drogas La Central, Droguería San Judas, Éticos. Lo propio ha hecho el Laboratorio del Caribe S.A. Estas cuestiones muestran el compromiso por parte del proveedor, a quien sin importar los costos en que muchas veces caiga, con el fin de aumentar la satisfacción en los clientes y generar lealtad, apuesta al fortalecimiento de las relaciones con los mismos, y estos, a su vez, han encontrado más valiosos los productos y servicios de sus proveedores y generan unos lazos tan estrechos que difícilmente lo cambiarán. Todo ello hace que esta relación perdure a lo largo del tiempo, pero en concreto lo que ha agregado valor a las droguerías ha sido la aplicación de estrategias tales como: 
Relación del proveedor de servicios farmacéuticos con sus clientes: Una mirada desde el Marketing Relacional en el departamento de Sucre

Servicios a domicilio, horario de atención extendido, acceso a internet, bases de datos de los clientes y acceso a discapacitados, tácticas que se han convertido en generadores de valor para las empresas en estudio.

No obstante lo anterior, otro de los resultados que arrojó la investigación cliente es que los canales de comunicación con el cliente por parte del proveedor, son insuficientes. Las droguerías visitadas no cuentan con una base completa de sus clientes, lo que no les permite generar una estrategia de fidelización con los mismos, puesto que todos los clientes no son iguales. Cabe aclarar que las droguerías objeto de investigación tienen estrategias promocionales; sin embargo, las promociones no siempre generan fidelidad en la clientela. Todo esto da como resultado unas deficientes relaciones proveedor-cliente, $\mathrm{y}$ en su defecto muy poca calidad en la relación de estos miembros.

Por otro lado, a partir del proceso de indagación, se constató que las droguerías han venido generado alianzas son sus distribuidores mayoristas, en la cadena de actores del sector farmacéutico. Esto último les ha permitido mantener un stock de inventario y surtido para atender oportunamente los requerimientos del cliente $\mathrm{y}$, por ende, ha propiciado la perdurabilidad de las farmacias en un mercado cada vez más agresivo.

Finalmente, a partir de la entrevista exploratoria, se percibió que los clientes se sienten seguros con los medicamentos y recomendaciones que le ofrecen los 
Relación del proveedor de servicios farmacéuticos con sus clientes: Una mirada desde el Marketing Relacional en el departamento de Sucre

encargados de las droguerías, dado el conocimiento y la experticia que manejan respecto al tema.

\section{DISCUSIÓN}

El sector farmacéutico colombiano y, por ende, el mercado ético cuenta con el mayor porcentaje de participación en el mercado total. Los productos de marca cuentan con el mayor valor de venta, pero los productos genéricos, ganan cada día mayor terreno en el mercado, y la gran mayoría de los productores son laboratorios nacionales (Vallejo \& Vallejo, 2008).

Los laboratorios de productos cosméticos del departamento de Sucre constituyen el 0,2\% del total nacional y los laboratorios de productos naturales ocupan el 2,2\% del total, de acuerdo con el último censo realizado por Global pharma (Sepúlveda, 2008). En este marco, la implementación de sistemas de control y adquisición de datos permitirá la estandarización de procesos en las empresas que se dedican a esta labor, así como cumplir exigencias de calidad, optimizando tiempos y costos.

Es evidente entonces que el cumplimiento de las exigencias del cliente, tales como: la agilidad y capacidad de respuesta por parte de los empleadores, la calidad en los productos y servicios, el mantenimiento de un contacto directo y continuo con ellos, la satisfacción de su necesidad, aun muchas veces sin poder conocerla, constituye uno de los pilares sobre los cuales se fundamenta el sector farmacéutico en Sucre, y que le permite crear ventaja competitiva con relación a los otros 
Relación del proveedor de servicios farmacéuticos con sus clientes: Una mirada desde el Marketing Relacional en el departamento de Sucre

departamentos de Colombia. De esto no se escapan algunos elementos adicionales, como la generación de reportes, el registro de datos, manejos de formulaciones, control de inventarios, reducción de duplicidad de información, todos los cuales se constituyen en la oportunidad de replantear los procesos de la organización y de obtener como beneficio la agilidad en la toma de decisiones a todos los niveles.

De acuerdo con el análisis, se constató con Vásquez - Velázquez (2005) los tres tipos de consumidores de medicamentos mencionados como resultado del estudio: i). los directos, en quienes predomina la soberanía en cuanto a preferencia y disponibilidad a pagar. ii). Los consumidores de medicamentos prescritos, cuyo pago se realiza como gasto de bolsillo, caso en el cual el médico actúa como agente, perfecto o imperfecto, de su paciente. iii). Los consumidores en los que prevalecen sistemas de seguridad social como agentes de los pacientes, caso en el que los medicamentos son prescritos y parte del pago es cubierto por el tercer pagador.

Sobre la base de las consideraciones anteriores, es posible enmarcar a las 285 droguerías inscritas en el departamento de Sucre, de un total de 14.208 existentes en Colombia, según lo expuesto por Sepúlveda (2008). Dichas droguerías son actores importantes en el sector farmacéutico en Sucre, dado que donde mayormente se desarrolla la actividad mercantil es en las droguerías. Así dado que el precio no juega un papel importante, por la estandarización propia 
Relación del proveedor de servicios farmacéuticos con sus clientes: Una mirada desde el Marketing Relacional en el departamento de Sucre

del sector, las relaciones que se establezcan jugarán un papel importante a lo largo del proceso.

Ahora bien para la identificación de calidad en la prestación del servicio, como uno de los aspectos más relevantes en la relación proveedor-cliente, se requiere analizar la manera como se deben desarrollar las relaciones. En este sentido, Dwyer, Schurr \& Oh (1987, apoyados en Scanzoni, 1979) exponen que las relaciones se amplían más allá del primer momento a través de cinco fases generales: (1) el conocimiento, (2) la exploración, (3) la expansión, (4) el compromiso, y (5) la disolución. Cada fase representa una transición importante en la forma como las partes se respetan una a otra, de modo que si una relación sobrevive a la fase de exploración y expansión, la transición al compromiso requiere un alto nivel de congruencia de objetivos o mecanismos de aplicación herméticos (Dwyer, Schurr and Oh 1987, p 22), dado que, a partir de allí, los procesos se hacen más complejos e inciertos. Estas fases en particular hacen hincapié sobre el rol de la calidad y en los intereses del comprador en el mantenimiento de una relación de calidad.

Walter et al (2001) establecieron que la calidad es fundamental en todos los aspectos de la relación, pues, permite que se genere valor en la organización y puede ser considerada como la razón de ser de las relaciones compradorproveedor. Su estudio identificó siete funciones en las relaciones con los clientes, tres de las cuales están directamente relacionadas con el 
Relación del proveedor de servicios farmacéuticos con sus clientes: Una mirada desde el Marketing Relacional en el departamento de

desempeño de una empresa: La función de volumen, que contribuye al éxito de la empresa, asegurando el "punto de equilibrio" necesario $\mathrm{y}$, por lo tanto, permite a la empresa operar en una base con fines de lucro; la función de beneficios de una relación de cliente, a partir de flujo de caja positivo dispuesto por el proveedor; y la función de salvaguardia que en un mercado dinámico se representa por la obtención de la estabilidad y el control en términos de ventas . Desde estas funciones, se puede observar como la calidad del servicio en la relación proveedor-cliente toma relevancia, permitiendo que a lo largo del tiempo se genere lealtad. Y esto, a su vez, genera valor, y produce en el cliente final la sensación de satisfacción, confianza y cooperación lo cual hace que las relaciones sean duraderas para las partes involucradas.

Las otras funciones desarrolladas por Walter et al son: la función de mercado, es decir, el acceso a nuevos mercados o clientes; la función de la innovación, que evidencia los conocimientos tecnológicos y las ideas creativas que se obtienen del cliente; la función de explorador, que propicia la obtención de información crítica a través de los clientes; y la función de acceso, que, como indica su nombre, permite el acceso a terceros y hace que esos actores sean accesibles, abiertos y comprensibles. Es de anotar que Walter et al (2001) precisan que estas últimas cuatro funciones no influyen directamente en el rendimiento de una empresa dentro de esa relación o en un momento determinado en 
Relación del proveedor de servicios farmacéuticos con sus clientes: Una mirada desde el Marketing Relacional en el departamento de

el tiempo, sin embargo, son importantes

para la calidad de la relación, pues permiten que la empresa se desarrolle en un futuro tanto a corto como a largo plazo.

El paradigma tradicional del marketing se caracteriza por una orientación de tipo transaccional. La tesis central al respecto se circunscribe a la mezcla de de cuatro variables que convencen al consumidor para que compre un determinado producto

(Gummesson, 2002). El marketing transaccional se limita a conseguir clientes y cerrar la venta, en un espacio de corto plazo, en el que se presenta un escaso contacto con el cliente final que no propende por la satisfacción total del cliente, sino solo por la transacción y ese orienta hacia el mercado (Bagozzi, 1975). pasivo y activo.

Córdoba López (2009) afirma que entre las debilidades del paradigma transaccional se destaca la fuerte orientación y concentración hacia la mezcla de marketing, que, mediante la apropiada mixtura de cuatro variables puede influir en el consumidor para que compre un determinado producto. Su orientación son las masas y el rol del proveedor y del cliente se en términos de

En contraposición a la teoría de marketing anterior, el marketing de relaciones trata de establecer, mantener y realzar las relaciones con los clientes y otros socios o partes del intercambio, de forma que los objetivos de las partes se encuentren (Grönroos, 1994). En este caso, el intercambio mutuo como parte 
Relación del proveedor de servicios farmacéuticos con sus clientes: Una mirada desde el Marketing Relacional en el departamento de Sucre

del cumplimiento de las promesas pondrá de manifiesto la búsqueda de beneficios para las partes involucradas, aspecto que se convierte en el pilar para mantener cada una de las relaciones que se establezcan.

El marketing de relaciones nació a partir de tres investigaciones en las que el cliente hace parte esencial: el IPM Group (Industrial and Internacional Marketing and Purchasing) (Hakansson, 1982), la Escuela Nórdica de Servicios (Berry y Parasuraman, 1993) y la economía de las relaciones con el consumidor (perspectiva comportamental) (Gómez et al, 2000). Cada una busca atraer al cliente (Berry, 1983), pero también mantener su vínculo con la organización, de manera que el cliente se vuelva parte de ella.
El IPM Group (Hakansson, 1982) basa sus estudios sobre el marketing en la perspectiva de redes de organizaciones, en las que sus miembros establecen relaciones cooperativas para obtener ventajas estratégicas significativas y la competencia se produce entre distintas redes (Aijo, 1996). La Escuela Nórdica de Servicios centra su atención en el marketing de servicios, que se basa en intercambios relacionales entre comprador y vendedor (Berry, Shostack y Upah, 1983, citados en Suárez et al, 2006).

Como objeto de estudio, el marketing de relaciones debe desarrollar una estrategia centrada en el cliente, esto es, en la integración de los canales de comunicación con los actuales y potenciales clientes, quienes contarán con 
Relación del proveedor de servicios farmacéuticos con sus clientes: Una mirada desde el Marketing Relacional en el departamento de Sucre

personal de “contacto” debidamente formado para identificar sus características. En realidad, se trata de una nueva forma de dirigir los negocios, que puede colocar a la organización en una posición dominante, convirtiéndose en una herramienta que permite maximizar el valor actual y el tiempo de vida de los clientes de la empresa, así como la maximización de la satisfacción de los clientes (Rajagopal \& Sánchez, 2005).

Morgan y Hunt (1994) añaden que la comprensión del marketing de relaciones requiere de la diferenciación entre la transacción discreta y el intercambio relacional, y clasifican las relaciones en alianzas o colaboraciones laterales (competidores, organizaciones no lucrativas, gobierno), internas (unidades de negocio, empleados y departamentos), con proveedores (de bienes y servicios) y con clientes, entre quienes se incluyen los intermediarios y los clientes finales (Sheth y Parvatiyar 1995; Gummesson, 1996; Alet, 2000). Según se ha citado, en el marketing de relaciones se reducen los lazos económicos, técnicos y sociales entre los miembros de la relación, es decir, entre el proveedor y el cliente, obteniéndose como beneficios adicionales el incremento de la confianza mutua, un mayor interés por ayudarse, la disminución de costos y la duración de las transacciones.

Hecha la observación anterior, se puede decir que el marketing de relaciones no es una teoría aislada. El hecho de depender del largo plazo hace 
Relación del proveedor de servicios farmacéuticos con sus clientes: Una mirada desde el Marketing Relacional en el departamento de

que reciba influencias de tres elementos esenciales: la tecnología de la información, el sector servicios y las nuevas estructuras de las empresas (Bejou, 1997). Respecto a esto último, resulta pertinente hacer énfasis en la notable evolución de las nuevas tecnologías de la información y las comunicaciones (NTIC) y en la forma cómo se han convertido en un factor determinante para el desarrollo del marketing de relaciones. Las tecnologías de la información (bases de datos relacionales, software de análisis, multimedia, entre otros) y los desarrollos en inteligencia artificial han abierto importantes expectativas que no existían en el pasado. El data warehouse, el data mining y la respuesta personalizada permiten a las organizaciones diseñar productos que cubren las necesidades esperadas de cada cliente.

Es evidente entonces que el comercio electrónico aporta una nueva dimensión al contacto a través de la alta tecnología, pero incide poco entre la relación de personas. El uso de tecnologías como CRM, Call Center, proyectos de fidelización Club clientes, o lo comúnmente conocido como tarjetas de puntos o tarjetas de cliente, no son otras cosas que estrategias para retener muy sutilmente al cliente. De la misma manera, los proyectos y la integración de sistemas de información que contribuyan al mejoramiento de las relaciones representan sólo una parte del marketing de relaciones, pero, en general, los expertos de marketing consideran que estas tecnologías pueden resolver 
Relación del proveedor de servicios farmacéuticos con sus clientes: Una mirada desde el Marketing Relacional en el departamento de Sucre

problemas y mantener la relación con el cliente.

Por todo lo expresado, el marketing de relaciones ha sido una de las aportaciones más importantes en el desarrollo de la moderna ciencia de marketing (Payne and Holt, 2001), de ahí que las investigaciones al respecto han puesto su punto de atención en las relaciones continuas, dejando de analizar exclusivamente los intercambios como sucesos o transacciones discretas en el tiempo.

Es necesario buscar alianzas y fortalecer no solo las relaciones con el cliente, sino también, en el caso de las droguerías, con sus distribuidores directos. En este sentido, Smith y Barclay (1997) afirman que el surgimiento de alianzas y asociaciones nuevas determinan la efectividad de estas relaciones y que la confianza es un ingrediente importante en el desempeño de las labores y en la satisfacción de las partes. Por esta razón, es preciso advertir que las percepciones de confianza se construyen con el tiempo, y que los proveedores deben aprender sobre los enfoques y las capacidades de cada cliente, lo cual se halla muy ligado a la calidad en términos de la relación, puesto que la satisfacción es como un cumplimiento placentero (Oliver, 1997, 1999). De este modo, tanto en el sector farmacéutico como en la mayoría de los sectores de la economía, la clave para la retención de los clientes está en satisfacer las necesidades de los mismos (Kotler, 1994, p. 20). 
Relación del proveedor de servicios farmacéuticos con sus clientes: Una mirada desde el Marketing Relacional en el departamento de Sucre

De acuerdo con nuestro estudio, es posible afirmar que, en el sector farmacéutico de Sucre, el modelo tradicional del marketing sigue siendo predominante. En este sentido, Pinto (1997) plantea la necesidad de que la empresa abandone su anterior orientación, limitada a promover transacciones, y se concentre en establecer relaciones, pues el reto ahora consiste en « ofrecer el valor conveniente, al cliente apropiado, en el momento adecuado» (p. 32). Adicionalmente, las empresas y organizaciones son cada vez más conscientes de la necesidad, en términos de rentabilidad, de retener clientes y no dedicar todos sus esfuerzos únicamente a conseguir clientes nuevos.

Entonces, el marketing de relaciones hace hincapié en la continuidad, mientras que el enfoque transaccional se fija en lo inmediato (Vásquez, 2000). Visto desde una perspectiva de intercambio transaccional, en este último caso, la relación con el cliente termina cuando se produce la venta, mientras que en el enfoque de intercambio relacional, con la venta apenas se da inicio a la relación con el cliente (Cuadro 1). Lo anterior significa pasar de lo tangible a lo intangible, de una estrategia de corto plazo a otra de largo plazo, es decir, que el objetivo de una organización ya no será a corto plazo, que se reduce a hacer la simple venta, lo tangible, y se convierte en un propósito a largo plazo, lo intangible, como es el crear a un cliente (Grönroos, 1996; Jüttner y Wehrli, 1994; Gummesson, 1997). 
Relación del proveedor de servicios farmacéuticos con sus clientes: Una mirada desde el Marketing Relacional en el departamento de Sucre

Con todo lo mencionado, es pertinente cuestionarse acerca del lugar en el que el marketing se vuelve tan relevante que traspasa la frontera de la transacción y se traduce en la creación de relaciones (Cavazos \& Giuliani, 2008), pues este nuevo paradigma trae consigo actores, recursos y actividades que pueden crear relaciones entre compradores, vendedores, accionistas y otros públicos, interesados en la posibilidad de que se pase de la transacción diádica (empresa-cliente) a las relaciones diádicas o incluso a organizar relaciones más complejas entre distintos actores (redes).

En otras palabras, mientras en el marketing de relaciones se crea valor en el "qué hacer por los clientes", en el marketing tradicional, la orientación está dada por "qué hacer a los clientes”.

Cuadro 1. Formas de intercambio

\begin{tabular}{|c|c|c|}
\hline \multirow{2}{*}{ Elementos Intercambio } & \multicolumn{2}{|c|}{ FORMAS DE INTERCAMBIO } \\
\hline & Transaccional & Relacional \\
\hline Objetivos & $\begin{array}{l}\text { Realizar una venta } \\
\text { Satisfacción necesidades del cliente }\end{array}$ & $\begin{array}{l}\text { Crear un cliente } \\
\text { Integración del cliente a través de la } \\
\text { creación interactiva de valor }\end{array}$ \\
\hline $\begin{array}{l}\text { Dimensiones temporales } \\
\text { - } \quad \text { Horizonte temporal } \\
\text { - } \quad \text { Naturaleza transacción }\end{array}$ & $\begin{array}{l}\text { Corto } \\
\text { Corta con principio y fin }\end{array}$ & $\begin{array}{l}\text { Extendido } \\
\text { La mayor duración }\end{array}$ \\
\hline 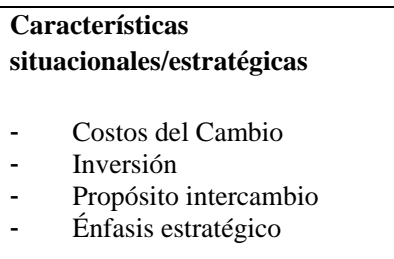 & $\begin{array}{l}\text { Bajos } \\
\text { Pequeña }\end{array}$ & $\begin{array}{l}\text { Altos } \\
\text { Alta }\end{array}$ \\
\hline
\end{tabular}


Relación del proveedor de servicios farmacéuticos con sus clientes: Una mirada desde el Marketing Relacional en el departamento de

Sucre

\begin{tabular}{|l|l|l|}
\hline & Económico & Sociales y Económicos \\
\hline Consecuencias & Bajo & Alto \\
$-\quad \begin{array}{l}\text { Complejidad } \\
\text { Reparto Beneficios y cargas }\end{array}$ & Simple & Compleja (interdisciplinaria) \\
& Fuerte división & División borrosa \\
\hline
\end{tabular}

Fuente: Adoptado de Gundlach y Murphy (1993) y Jûttner y Wehru (1994).

Cuadro 2. Diferencias entre el marketing transaccional y el marketing de relaciones

\begin{tabular}{|l|ll|}
\hline \multirow{4}{*}{ MARKETING TRANSACCIONAL } & - & Se centra en las ventas individuales \\
& - & Resalta las características de los productos, no los beneficios \\
& - & Planifica a corto plazo \\
& - & Poco énfasis en la atención y satisfacción del cliente \\
& - & Nivel moderado de contactos con el cliente \\
& - & La calidad se centra, casi exclusivamente, en el producto \\
\hline & - & Considera a los clientes el activo más importante de la empresa \\
& - & Énfasis en retener y satisfacer clientes \\
MARKETING DE RELACIONES & - & Resalta los beneficios del producto \\
& - & Planifica y posee visión a largo plazo \\
& - & Alto nivel de compromiso y de contacto con los clientes \\
& & La calidad como algo que incumbe a toda la organización \\
\hline
\end{tabular}

Fuente: Adoptado de Christopher, Payne y Ballantyne (1991).

El marketing de relaciones está basado en un proceso en el que la empresa construye relaciones a largo plazo con clientes actuales $y$ potenciales, $y$ en el que comprador y vendedor trabajan de manera conjunta hacia la consecución de unos objetivos comunes (Evans y Laskin,

1994, p. 40). Las aplicaciones del marketing relacional se enfocan en temas como la calidad, la calidad de servicio, la lealtad del cliente, el valor, la calidad de la relación y la orientación al mercado. 
Relación del proveedor de servicios farmacéuticos con sus clientes: Una mirada desde el Marketing Relacional en el departamento de Sucre

De hecho, cada día estamos más involucrados en el trato continuo con personas, y esto obliga a las organizaciones a formarse en una universalidad de pensamientos, en un ambiente globalizado, donde la excelencia en el servicio al cliente constituye un valor agregado y diferenciador entre organizaciones (Hayes, 2000).

Hoffman \& Bateson (2002) señalan en este sentido que un servicio de calidad implica: clientes y el de la calidad de los servicios están entrelazados. En consecuencia, la calidad de los servicios no es una meta específica ni un programa que se cumpla o termine, sino que debe ser una parte permanente de toda la administración o producción de servicios, todos los días. (pp. 325, 326)
Actitud debida a una evaluación global, a largo plazo, del desempeño de una empresa. No cabe duda de que el concepto de la satisfacción de los
Cabe anotar que los estudios formales de calidad del servicio tuvieron sus inicios con los trabajos de Parasuraman, Zeithaml y Berry (1985). En ellos se destacaba que los servicios presentan una mayor problemática para su estudio, pues poseen características que crean una gran 
Relación del proveedor de servicios farmacéuticos con sus clientes: Una mirada desde el Marketing Relacional en el departamento de

brecha y que los diferencian ampliamente de los productos, tales como la intangibilidad, la heterogeneidad y la inseparabilidad En concordancia con los anteriores autores, Duque (2005) presenta estas características como diferenciadores entre productos y servicio:

- Intangibilidad: Los servicios no son objetos, y esto permite aseverar que el cliente no puede asegurarse de la calidad antes de su compra.

- Heterogeneidad: Los resultados de la prestación del servicio son variables, puesto que cada cliente percibe la calidad a su manera. En ocasiones, incluso, de forma muy diferente a como la empresa la percibe, ningún cliente es igual a otro. Así, es difícil generar una calidad uniforme, porque lo que la empresa cree prestar puede ser diferente de lo que el cliente percibe.

- Inseparabilidad: En muchos servicios, la producción y el consumo son indisociables (Grönroos, 1978). En servicios intensivos en capital humano, a menudo tiene lugar una interacción entre el cliente y la persona de contacto de la empresa de servicios que afecta considerablemente la calidad y su evaluación.

En todo caso, en la mayoría de las ocasiones, un servicio con calidad conlleva a la lealtad del cliente hacia la empresa (Müller de la Lama, 2003). Para un cliente, no es una tarea fácil evaluar la calidad del servicio que se le ofrece. Por tal razón, la percepción de la calidad del servicio sería el resultado de una comparación del cliente con el 
Relación del proveedor de servicios farmacéuticos con sus clientes: Una mirada desde el Marketing Relacional en el departamento de Sucre

desempeño actual del servicio. No obstante, en ocasiones, los proveedores no comprenden que los clientes son las personas más importantes de un negocio, que no son un simple objetivo en el trabajo sino un fundamento (Cervantes \& Steffanell 2011) y que estos perciben la calidad en los servicios a partir de sus propias expectativas (Grönroos 1988). Por ello, un proveedor que suele tener dificultades para comprender cómo perciben sus clientes la calidad de los servicios que presta (Zeithaml, 1981).

Según Spreng \& Mackoy (1996), la calidad en el servicio es un constructo distinto a la satisfacción, pero también un antecedente importante de este último y del cual dependen de alguna manera los resultados de la organización. Los autores antes mencionados determinaron empíricamente que los atributos de la calidad en el servicio se separan estadísticamente de la satisfacción cuando esta última se mide mediante la di confirmación de expectativas. Los atributos de la calidad en el servicio también se han establecido como antecedente de la lealtad hacia la marca. En diversos estudios, se ha encontrado que tales atributos tienden a presentar relaciones estadísticas positivas con diversas mediciones de lealtad como: intención de compra, disposición a cambiar de marca, disposición a pagar más por la marca, y en algunos casos acción de re-compra (Chao, 2008; Fullerton \& Taylor, 2002; Devaraj et al, 2001).

Este concepto de las relaciones comprador-vendedor se ha aplicado a 
Relación del proveedor de servicios farmacéuticos con sus clientes: Una mirada desde el Marketing Relacional en el departamento de

diferentes niveles de los canales de marketing (proveedor-fabricantedistribuidor-cliente), y es claro que un cliente es leal a marcas de productos y/o servicios que satisfagan sus necesidades. Al cumplirse esto, el cliente puede atraer a otros, forjando relaciones que se harán más estables a lo largo del tiempo y permitirán a la organización permanecer en este ambiente cada vez más globalizado. En cambio, algunos estudios muestran que la insatisfacción de los clientes con el servicio que se les presta conlleva la pérdida del cliente y del círculo de relaciones en el que este se mueve (Hochman, 2005; Technical Assitance Research Program (TARP). El mismo Hochman (2005) sostiene que por cada cliente insatisfecho se pierden 30 clientes potenciales.
En el mismo orden y dirección, la calidad de la relación ha sido definida como «el grado de conveniencia que tiene una relación para un cliente a la hora de satisfacer sus necesidades» (HennigThurau y Klee, 1997, p. 751). Y en este mismo sentido, Gummesson (1987) considera la calidad de la relación como la calidad de la interacción entre un cliente y un proveedor, y puede ser interpretada en términos de valor acumulado. Para comprender el constructo de calidad de la relación, se requiere considerar las definiciones del constructo en su conjunto, es decir, partir de sus atributos y sus consecuencias (Roberts et al, 2003), basado en Bagozzi, 1984) así como de las evaluaciones de las dimensiones que constituyen el constructo. 
Relación del proveedor de servicios farmacéuticos con sus clientes: Una mirada desde el Marketing Relacional en el departamento de

De acuerdo con el análisis de las definiciones de los atributos, la visión más reciente de la calidad de la relación postula que este es un constructo de orden superior, compuesto por dimensiones diferentes pero relacionadas (Hibbard et al, 2001; Hewett et al, 2002; Roberts et al, 2003; Hennig et al, 2002; Lages et al, 2005; Ulaga y Eggert, 2006). Crosby, Evans y Cowles (1990) afirman al respecto que cuando el cliente es capaz de confiar en la integridad del proveedor y confía en su desempeño futuro es porque el nivel de desempeño pasado ha sido coherentemente satisfactorio. Estos autores, concluyeron lo anterior a partir de pruebas empíricas de un modelo de calidad de la relación que confirmó la asociación positiva entre la calidad de la relación y la intención de compra, y la existencia de al menos dos dimensiones: la confianza y la satisfacción.

Un cliente no será fiel a su proveedor si este no le ofrece confianza. De esta manera, cuando existe una completa satisfacción en la relación proveedor-cliente, el beneficio mutuo se refleja en la calidad de la relación. Las aproximaciones de algunos autores, a partir de sus investigaciones, concuerdan en que la satisfacción del cliente con la actuación de un proveedor, la confianza con ese proveedor, y el compromiso del cliente son las variables que fundamentan la calidad de la relación (Baker et al, 1999; Dorsch al, 1998; Garbarino y Johnson, 1999; Hewett et al, 2002; Smith, 1998; Roberts et al, 2003; Ulaga y Eggert, 2006). 
Relación del proveedor de servicios farmacéuticos con sus clientes: Una mirada desde el Marketing Relacional en el departamento de Sucre

Acorde con lo antes dicho, en nuestro estudio del sector farmacéutico, se encontró que la perduración de la relación requiere que quien ofrezca el servicio inspire confianza y que el cliente tenga certeza de que el producto ofrecido aliviará su padecimiento. Crosby et al (1990) confirman en este sentido que la satisfacción y la confianza son componente clave de la calidad de la relación. Igualmente, Dorsch et al (1998) aseveran que la calidad de la relación está indicada por la confianza, el compromiso y la satisfacción por parte de los compradores.

Algunos académicos $\quad \mathrm{y}$ profesionales buscan medir con rigor la calidad percibida, con el fin de entender al cliente en su integralidad y satisfacer, de esta manera, sus necesidades. Si bien esto es importante, lo que se debe buscar ante todo son métodos para mejorar la calidad, a fin de que la organización logre una ventaja competitiva basada en la lealtad que el cliente manifieste no solo hacia el producto sino hacia la organización (Palmer y Berou, 1995; Zahorik y Rust, 1992).

Es oportuno referirse a Cantú (1999), para quien los principales factores de calidad son el comportamiento, la actitud y la habilidad del servidor humano, y que los errores cometidos se presentan durante la prestación, el tiempo de espera y el tiempo de entrega del servicio. Se observa claramente entonces que este es uno de los factores que deben fortalecer todos los sectores de la economía, y en este estudio en particular, el sector farmacéutico, puesto que la 
Relación del proveedor de servicios farmacéuticos con sus clientes: Una mirada desde el Marketing Relacional en el departamento de Sucre

satisfacción se explica en gran medida por la calidad percibida.

Ahora bien, como acaso se ha reiterado, la satisfacción es un concepto subjetivo, que depende de las percepciones de los clientes, y se deriva de un proceso entre las expectativas que poseen frente al producto y el desempeño real de este, que solo se conoce una vez adquirido (Oliver, 1980; Bei \& Chiao, 2001). Por esto, la satisfacción es una dimensión que solo tiene dos posibilidades extremas: el cliente puede mostrarse satisfecho frente a los beneficios obtenidos o, por el contrario, manifestar su inconformidad.

De la misma manera, la satisfacción se define como una respuesta afectiva frente a un producto o servicio obtenido, que se deriva de la comparación del resultado de un producto con el servicio (Halstead et al, 1994) en el que se tienen criterios y rangos de valoración que se han alcanzado en compras anteriores, y se establecen como paradigmas guías que se fortalecen o desaparecen a medida que se ejercen procesos de compras.

Por consiguiente, la satisfacción se relaciona tanto con la experiencia de compra como con la experiencia de consumo, es decir, con el servicio ofrecido y con el producto adquirido. Por lo tanto, es fundamental diferenciar la satisfacción con el producto y la satisfacción con el proveedor, ya que si se pueden conocer las inconformidades del cliente frente al proceso a partir del cual le fue concedido el material deseado, se facilita una evaluación paulatina del 
Relación del proveedor de servicios farmacéuticos con sus clientes: Una mirada desde el Marketing Relacional en el departamento de

itinerario de compra que debe llevar a cabo un comprador. Las ventajas de concebir la satisfacción desde estos dos puntos de vista radica en que permite que las relaciones entre comprador-proveedor sean integrales, es decir, que se dé la oportunidad de evaluar no solo la satisfacción frente a los beneficios del producto, sino también la comodidad o inconformidad con el proceso vivido para adquirir el material objeto de compra. Por ejemplo, si el comprador está satisfecho con los beneficios del producto, pero no con el servicio ofrecido, el proveedor puede cambiar su estrategia de distribución y venta; en caso contrario, al presentarse insatisfacción con el producto, se analiza y se evalúan posibles cambios para alcanzar un nivel de calidad
Cuando un cliente se siente satisfecho, se crea un puente para generar confianza y afianzar bases para consolidar una relación comercial sólida en la que se garantice su compromiso con el proveedor y la permanencia de la relación. Es importante, en consecuencia, conocer los distintos conceptos que varios autores han construido sobre satisfacción: Oliver (1981, p. 27) la definió como "una evaluación de la sorpresa inherente en una adquisición de productos y / o experiencia de consumo", entrando en la dimensión afectiva. El mismo Oliver y Mano (1993) la entienden conceptual y empíricamente como un enlace que ocurre de forma natural, y en el que se combinan los juicios cognitivos y las reacciones afectivas. mayor. 
Relación del proveedor de servicios farmacéuticos con sus clientes: Una mirada desde el Marketing Relacional en el departamento de Sucre

Para Tse, Nicosia y Wilton (1990), la satisfacción del consumidor es un proceso subjetivo, multidimensional $\mathrm{y}$ dinámico que sigue al acto de compra. Por último, Ravald y Grönroos (1996), así como Liljander y Strandvik (1995) consideran que la satisfacción del cliente es un fuerte predictor de variables de comportamiento, tales como las intenciones de recompra, el boca-a-boca o la lealtad . A partir de lo anterior, el concepto de satisfacción se enmarca dentro de un contexto en el que priman la dimensión afectiva, y las expectativas frente a y lo que se obtiene después de, lo que la convierte en uno de los pilares del progreso, pues de ella dependen las relaciones comerciales y su viabilidad.

No se debe olvidar la necesidad de generar estímulos para satisfacer al cliente, trabajo en el que se deben tener en cuenta detalles como: preferencias más comunes y las inconformidades a partir de las cuales se puede mejorar las relaciones de satisfacción entre proveedor y cliente. Finalmente, en la relación con el cliente, es necesario fortalecer la lealtad, pues esta genera una serie de efectos económicos que se propagan en cascada en los diferentes niveles de la compañía (Valencia, 2007). Los ingresos y la participación de mercado crecen cuando los clientes más rentables son atraídos a la empresa, creando ventas repetitivas y referencias favorables en otros clientes. De este modo, la organización puede concentrar sus inversiones en los segmentos de clientes más rentables y potencialmente leales, generando un crecimiento sostenible para la organización, lo que, visto desde otro 
Relación del proveedor de servicios farmacéuticos con sus clientes: Una mirada desde el Marketing Relacional en el departamento de Sucre

ángulo, no es más que una cadena de

en

la

Figura

1

lealtad con el cliente, como se representa

Figura 1. Cadena de lealtad

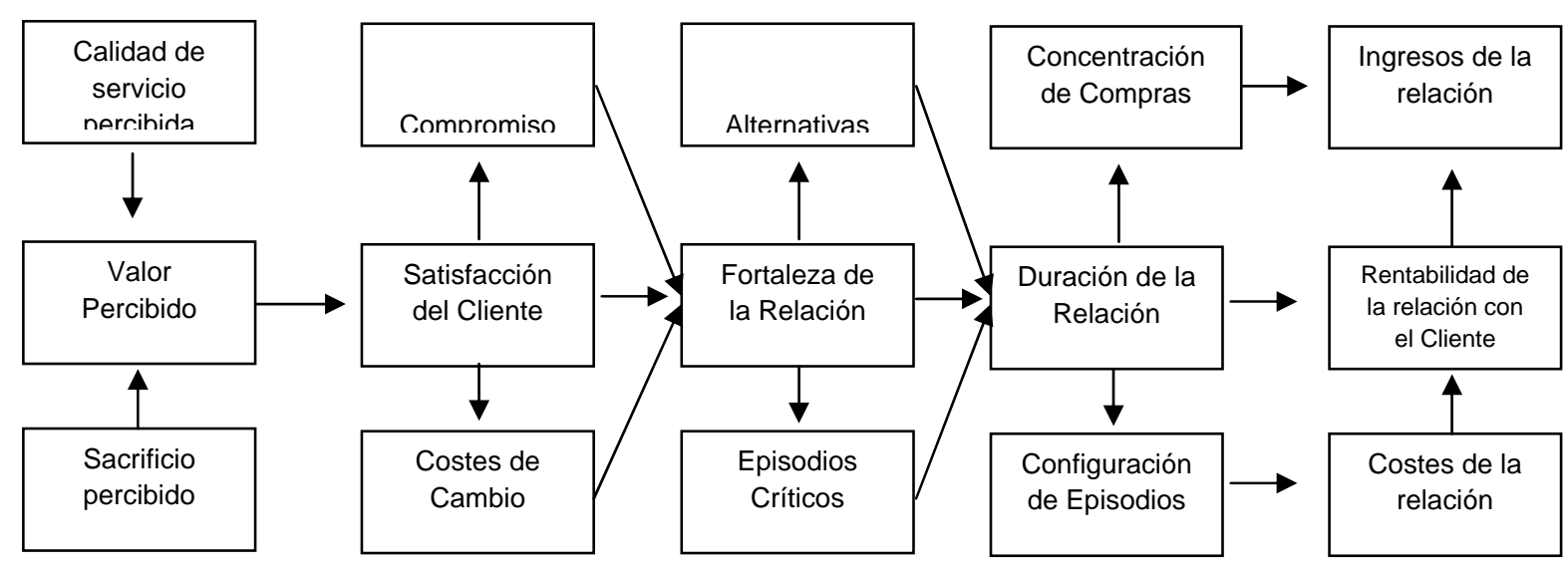

Fuente: Storbacka et al (1994, adoptada de Barroso Castro y Martín Armario, 1999).

\section{REFERENCIAS}

Aijo, T. S. (1996). The theoretical and philosophical underpinnings of relationship marketing. Environmental factors behind the changing marketing paradigm. En: European Journal of Marketing, 30 (2), 8-18.

Alet, J. (2000). Marketing relacional. Barcelona: Ediciones Gestión.
Bagozzi, R. P. (1984). A prospectus for theory construction in marketing, Journal of Marketing, (48), 11-29.

Bagozzi, R. (1975). Marketing as exchange. Journal of Marketing. (39)

Baker, TH. L.; Simpson, P. M. y Siguaw, J. A. (1999). The Impact of Suppliers Perceptions of Reseller Market Orientation on Key Relationship 
Constructs», Journal of the Academy of Marketing Science, 27 (1), 50-57. case of service marketing. Journal of Retailing, 69 (1): 16-3
Bei, L. \& Y. Chiao (2001). An integrated product, perceived service quality, and perceived price fairness on consumer satisfaction and loyalty. Journal of consumer satisfaction, dissatisfaction, and complaining behavior, (4), 126-140.

Berry, L. L. (1983). Relationship marketing. L. L. Berry, G. L. Shostack y G. D. Upah (Editors). Emerging perspectives of service marketing. Chicago: American Marketing Association.

Berry, L. L. \& Parasuraman, a (1993). Building a new academic field - the

Bejou, D. (1997). Relationship marketing: Evolution, present state, and future. Psychology \& Marketing. New Jersey, (14), 727-735. model for the effects of perceived

Cantú, H. (1999). Desarrollo de una cultura de calidad. Madrid: McGrawHill.

Cavazos, J. \& Giuliani A. (2008). El concepto de marketing bajo el paradigma relacional una agenda para Latinoamérica. Invention Argentina. 11 (20) 41-51.

Chao, P. 2008. Exploring the nature of the relationships between service quality and customer loyalty: an attributelevel analysis. The Service Industries Journal, 28 (1), 95-115.

Cervantes Atia, V. \& Steffanell, Santiago, I. (2011). Modelo de gestión en el servicio como herramienta de competitividad en una institución de educación superior. Revista Desarrollo Gerencial 3(2), 273-200 Universidad Simón Bolívar. Colombia. 
Córdoba López, J. F. (2009). Del Production and Operations marketing transaccional al marketing relacional. Entramado. Cali, Colombia. (9), 6-17

Concha, J.R. y Solikova, A. (2000). Relaciones a largo plazo entre compradores y proveedores: La importancia de la tecnología en el desarrollo de la confianza. Estudios Gerencial, (76), 13-25.

Management, 10 (4), 424-439.

Dorsch, M. J.; Swanson, S. R. y Kelley, S. W. (1998). The Role of relationship quality in the stratification of vendors as perceived by customers. Journal of the Academy of Marketing Science, 26(2), 128-142.

Duque, E-J. (2005). Revisión del concepto de calidad del servicio y sus modelos de medición. Innovar. Revista de Ciencias Administrativas y (1990). Relationship quality in services selling: An interpersonal influence. Perspective Journal of Marketing, 54, 68-81.

Dwyer, R. Schurr, P. and Oh, S. (1987). Relationships. Journal of Marketing. (51) $11-27$.

Evans, J. R. y Laskin, R. L. (1994). The relationship marketing process: A Conceptualization and Application. Industrial Marketing Management, (23): 439-452.

Devaraj, S., K. F. Matta, \& E. Conlon Fullerton, G. \& Taylor, S. (2002). (2001). Product and service quality: Mediating, interactive and nonlinear the antecedents of customer loyalty in the automotive industry. effects in service quality and satisfaction with service research. 
Canadian Journal of Administrative

Science, 19 (2), 124-136.
Grönroos, C. (1996) “Relationship Marketing: strategic and tactical implications”. Management Decisions, 34(3), 5-14.

Garbarino, E. y Johnson, M. K. (1999).

The different roles of satisfaction, trust and commitment in customer relationships. Journal of Marketing, (63), 70-87.

$$
\begin{aligned}
& \text { Grönroos, C. (1994) "From Marketing } \\
& \text { Mix to Relationship Marketing: } \\
& \text { towards a paradigm shift in } \\
& \text { marketing", Management Decision, } \\
& \text { 32(12), 4-20. }
\end{aligned}
$$

Gómez, J. T.; Bello, L y Cervantes, M (2000). Del marketing de relaciones al marketing postmoderno. Revista Europea de Dirección y economía de la empresa. 9 (3), 113-124.

Gummesson, E. (1987), »The New Marketing-Developing Long-Term Interactive Relationships», Long Range Planning, 20(4), 10-20.

Grönroos, C. (1978). A Service Oriented Approach to Marketing of Services. European Journal of Marketing, 12(8), 588-601.

Gummesson, E. (1996): Relationship marketing and imaginary organizations: a synthesis. European Journal of Marketing, 30 (2), 31-44.

Grönroos (1988). Service Quality: The Six Criteria of Good Service Quality. Review of Business. New York: St. John’s University Press.

Gummesson, E. (1997). Relationship Marketing as a Paradigm Shift: Some Conclusions from the $30 \mathrm{R}$ Approach”, Management Decision, 35 (3) (4), 267 - 273. 
Gummesson, E. (2002) Total Relationship Marketing. Second Edition ed. Oxford: ButterworthHeinemann.

Gundlach, G.T. \& Murphy, P. E. (1993): «Ethical and Legal Foundations of Relational Marketing exchanges». Journal of Marketing, (57) (October), 35-46

Hakansson. H (1982): An interaction approach. In Hakansson. University of Uppsala (Ed) International Marketing and purchasing of industrial goods: an interaction. Chichester: John wiley \& sons, 1026.

Halstead, D., D. Hartman \& S. Schmidt (1994).Multisource effects on the satisfaction formation process. Academy of Marketing Science Journal, 22(2), 114-129.
Hayes, Bob E. (2000). Como medir la satisfacción del cliente: desarrollo y utilización de cuestionarios. Barcelona: Gestión,

Hennig-Thurau, Th. y Klee, A. U. (1997), «The Impact of Customer Satisfaction and Relationship Quality on Customer Retention: A Critical Reassessment and Model Development», Psychology \& Marketing, 14(8), 737- 765.

Hennig-Thurau, Th.; Gwinner, K. P. y Gremler, D. D. (2002), «An Integration of Relational Benefits and Relationship Quality», Journal of Service Research, 4(3), .230-247.

Hewett, K.; Money, R. B. y Sharma, S. (2002), «An exploration of the moderating role of buyer corporate culture in industrial buyer-seller relationships», Journal of the Academy of Marketing Science, 30(3), 229-239. 
Hibbard, J. D.; Kumar, N. y Stern, L. M. (2001), «Examining the impact of destructive acts in marketing channel relationships», Journal of Marketing Research, (38), 45-61.

Hoffman \& Bateson (2002). Fundamentos de marketing de servicios. $2^{a}$ edición. México: Thomson.
Kotler, P. (1994), Marketing Management. NJ. Prentice Hall.

Lages, C.; Lages, C. R. y Lages, L. F. (2005), «The RELQUAL scale: a measure of relationship quality in export market ventures», Journal of Business Research, (58), 1040-1048.

Liljander, V. y Strandvik, T. (1995), «The

Jüttner, U.; Wehrli, H. P. (1994): nature of customer relationship on "Relationship Marketing from a Value System Perspective”, International Journal of Service Industry Management, 5(5), 54 - 73. services », en Swartz, T.A.; Bowen, D.E. y Brown, S.W. (Eds), Advances in Services Marketing and Management: Research and Practice, (4), JAI Pres, Inc., Greenwich, CT, 141-167.

Koeting, J. R. (1984) Foundations of naturalistic inquiry: developing a theory base for understanding individual interpretations of reality. Dallas: Association for Educational Communications and Technology.

Llorens, J., Fandos, J.C., Moliner, M.Á. \& Sánchez, J.(2006). La calidad de la relación en mercados industriales: estado de la cuestión. Innovar 16(28), 199-222. 
Oliver, R. L. (1980). A cognitive model of the antecedents and consequences of satisfaction decisions. Journal of Marketing Research, 17(4), .460-469.

Oliver, R.L. (1981). Measurement and evaluation of satisfaction processes in retailing settings. Journal of Retailing. 57(3), 25-48.

Oliver (1997), Satisfaction: A Behavioral Perspective on the Customer, New York: McGraw-Hill.

Mano, H., Oliver, R.L. (1993). Assessing the dimensionality and structure of the consumption experience: evaluation, feeling and satisfaction. Journal of Consumer Research, 20(1), 451-466, dezembro

Morgan, R. M. y Hunt, S. D. (1994): The commitment-trust theory of relationship marketing. Journal of Marketing, (58), 20-38.
Müller de la Lama. (2003). Cultura de calidad de servicio. Mexico: Trillas.

Palmer, A. Bejou D. (1995), "The effects of gender on the development of relationships between clients and financial advisers," The International Journal of Bank Marketing, 13 (3), 18.

Payne, A. and Holt, S. (2001). "Diagnosing Costumer Value: Integrating the value process and Relationship Marketing” British Journal of Management. (12). 159182.

Pinto, Stephen K. (1997): «Marketing de relación o la transformación de la función de marketing». Harvard Deusto Business Review， 32-40

Quivy, Raymond y Van Campenhoudt, Luc. (2006). Manual de investigación en ciencias sociales, Las etapas del procedimiento, Limusa Grupo 
Noriega: Buenos Aires, Prometeo Libros.

Ramírez, Arcila, Buriticá, y Castrillón (2004). Paradigmas y modelos de investigación guía didáctica $\mathrm{y}$ módulo. Medellín Colombia: Fundación Universitaria Luis Amigo. Facultad de Educación. 2 ediciones

Ravald, A. and Grönroos, C. (1996): The Valúe Concept and Relationship Marketing, European Journal of Marketing 30(2):19-30

Rajagopal \& Sanchez, R. (2005), Analysis of Customer Portfolio and Relationship Management Models: Bridging Managerial Gaps, Journal of Business and Industrial Marketing, 20 (6), 307-316

Roberts, K.; Varki, S. y Brodie, R. (2003), «Measuring the quality of relationships in consumer services: an empirical study», European Journal of Marketing, (37). 169-196
Sepúlveda J. (2008) Caracterización Ocupacional del Sector Farmacéutico en Colombia, enfoque por entornos. Medellin: Servicio Nacional de Aprendizaje. SENA. Colombia.

Sheth, J. N. \&Parvatiyar, A. (1995): Relationship marketing in consumers markets: antecedents and consequences. Journal of the Academy of Marketing Science, 23(4), 255-271.

Storbacka, K., Strandvik, T. y Grönroos, C. (1994). "Managing Customer Relationships for Profit: The Dynamics of Relationship Quality “. International Journal of Service Industry Management, (5), 21-38

Smith, J. B. (1998), «Buyer-Seller Relationships: Similarity, Relationship Management, and Quality», Psychology \& Marketing, vol. 15(1), 3-21. 
Spreng, R. \& R. Mackoy (1996). An empirical examination of a model of perceived service quality and satisfaction. Journal of Retailing, 72(2), 201-214.

Suárez, L. Álvarez; Vázquez, R. Casielles; Díaz, A. Ma Martín. (2006) Factores determinantes de las relaciones estables entre una agencia de viajes y diversos tipos de clientes: consecuencias sobre el comportamiento. Cuadernos de Economía y Dirección de la Empresa. (29), 193-228.

Tse, D. \&Wilton, P. (1988). Models of consumer satisfaction formation: An extension. Journal of Marketing Research (25), 204-212.
Valencia, J. (2007). Fidelización y lealtad como estrategias para impactar favorablemente el recaudo y la cartera de las empresas. El Cuaderno

- Escuela de Ciencias Estratégicas 2(3), 11-22 Medellín - Colombia.

Vallejo, B.M., Vallejo, S.B. (2008). Aspectos generales de la automatización industrial del sector farmacéutico. Revista Colombiana de Ciencias Químicas Farmacéuticas. 35 (1), 47-63
Vázquez, R.; Díaz, A. Mª \& Del Rio, A. (2000), "El proceso de desarrollo de las relaciones comerciales entre comprador y vendedor", Área de Comercialización e Investigación de Mercados, Universidad Oviedo España.

Ulaga, W. y Eggert, A. (2006), «Relationship value and relationship quality: Broadening the nomological network of business-to-business relationships», European Journal of Marketing, 40(3/4), 311-327. 
Vásquez Velásquez, Joanna (2005). El sector farmacéutico en el marco de las negociaciones del tratado de libre comercio con los Estados Unidos. Perfil de Coyuntura Económica, (5), 33-43, Universidad de Antioquia. Colombia

Walter, A., Ritter, T., \& Gemünden, H. G. (2001). Value creation in buyer-seller relationships: theoretical considerations and empirical results from a supplier's perspective. Industrial Marketing Management, 30(4), 365-377.

Zahorik, A. J. and R.T. Rust Eds. (1992), modeling the impact of service quality on profitability: A review: JAI Press.

Zeithalm, V.A. (1981). How Consumer Evaluation Processes Differ between. Goods and Services. Marketing of Services, Chicago: American Marketing Association. 